[2] Beresniak A, Baerwald C, Zeidler H et al. Clin Exp Rheumatol. 2013 May-Jun;31(3):400-8.

Disclosure of Interest: A. Neubauer Grant/research support from: BMS, Intercept, Roche, Santen, C. Minartz Grant/research support from: BMS, Intercept, Roche, K. Herrmann Employee of: BMS, R. Postema Employee of: BMS, C. Baerwald Grant/research support from: Abbvie, Biogen, BMS, Chugai, Hexal, Medac, Pfizer, Roche, Sanofi Aventis, UCB DOI: 10.1136/annrheumdis-2017-eular.4312

\section{THU0627 TREATMENT OF RHEUMATOID ARTHRITIS: ADHERENCE TO GUIDELINES IN PRIVATE PRACTICE}

L.B. Fernandes, F.A. Palacios, P.G. Lerner, C.G. Macedo, F. Omura, E.A.K. Azeka, I.M.M. Laurindo, P. Toth, R.C. Ferreira, R.A.O. Aurichio, A.P. Bazilio, J. Brudniewski, A.L. Hayata. Private Office, São Paulo, Brazil

Background: Guidelines and therapeutic strategies in the treatment of rheumatoid arthritis (RA) have been developed and adopted by most Societies of Rheumatology. However, the extent to which they have been supported by rheumatologists in their individual clinical practice is unclear.

Objectives: Our aim was to analyze, at private offices, adherence to guidelines and characteristics of RA patients in regular follow-up.

Methods: This was a cross-sectional study developed by a group of rheumatologists $(n=13)$ working exclusively in private offices and hospitals in São Paulo, Brazil. It consisted of a web-based questionnaire addressing patient's demographics, social characteristics and treatment. Patients having the diagnosis of RA should be included sequentially. As Brazil's private health has no reference flowchart, patients can consult any physician from their insurance health program, or any physician at all if bearing the costs. Insurance programs can be either personal or tied to employers, in which case job changes imply in insurance and health professional changes.

Results: Data from 235 RA patients were collected, 84\% were female, mean age (SD) 57.3 (13.3) yrs., disease duration 9.5 (1-54) yrs., 73\% RF positive (74\% of the 61 RF negative patients had an anti-CCP test and 8 were positive), The mean duration of symptoms until diagnosis was $1.7 \mathrm{yrs}(0-23)$, number of physicians until diagnosis 2.4 ( 1 - 12), including GP (102 patients), orthopedists (110 patients) and rheumatologists (129 patients). Even after diagnosis, patients switched professionals at a mean number of 2 physicians (1-6), justified by shift of health insurance (66 patients), lack of resolution (33 patients), dislike of the physician (26 patients) and cost (11 patients). Regarding treatment, $51 \%$ of patients were on biologic agents: $23 \%$ adalimumab, 15\% abatacept and rituximab, $14 \%$ etanercept, $12 \%$ tocilizumab, $7.6 \%$ golimumab, $5 \%$ certolizumab and infliximab and $4 \%$ tofacitinib. Interestingly, most patients (75\%) using abatacept, tofacitinib and tocilizumab were not on methotrexate (MTX), while from the 65 patients on an anti-TNF agent, $75 \%$ were also using MTX or leflunomide (LFN). Regarding traditional DMARDs, $91 \%$ of patients were on $(n=94)$ or had used MTX $(n=120)$; $43 \%$ were on $(n=37)$ or had used $(n=64)$ LFN. Concerning treatment and disease activity, $91 \%$ of patients were considered adherent to the treatment with a mean number of 4 annual visits, $82 \%$ were also deemed to be in regular monitoring. According to rheumatologist's assessment, $63 \%$ of patients had RA "under control". In fact, $72 \%$ of patients were on low disease activity (22\%) or on remission $(50 \%)$, according to the DAS28. However, when patients in disease activity were analyzed, few of them $(0.28 \mathrm{Cl} 0.18-0.40)$ were on biologic or target DMARDS, pointing to a possible therapeutic transitional moment. Only 3 of these could be labeled "refractory", having previously used 3 biologic agents.

Conclusions: Treat-to-target strategy seems to have been adopted by most rheumatologists in their individual practice, although there is room for improvement and optimization of therapy. While treatment guidelines are roughly followed, the delay in diagnosis and changes of healthcare are particularly worrisome and need to be addressed.

Disclosure of Interest: None declared

DOI: 10.1136/annrheumdis-2017-eular.5521

\section{THU0628 PATTERNS OF ETANERCEPT DOSE ADJUSTMENTS IN A REAL-WORLD SETTING: A CANADIAN RETROSPECTIVE COHORT STUDY}

M. Khraishi ${ }^{1}$, Y. Zhang ${ }^{2}$, J. Ivanovic ${ }^{2}$, B. Millson ${ }^{2}$, E. Singh ${ }^{3}$, J. Woolcott $^{4}$ H. Jones ${ }^{3}$. ${ }^{1}$ Faculty of Medicine, Memorial University of Newfoundland, St. Johns: ${ }^{2}$ Health Access \& Outcomes, QuintilesIMS, Kanata, Canada;

${ }^{3}$ Inflammation and Immunology, Global Medical Affairs, Pfizer Inc., Collegeville, United States; ${ }^{4}$ Health Economics and Outcomes Research, Pfizer Inc., Kirkland, Canada

Background: Etanercept is a soluble TNF receptor (humanized protein) indicated for treatment of immune-mediated inflammatory diseases, including rheumatoid arthritis (RA), psoriatic arthritis (PsA), psoriasis (PsO), and ankylosing spondylitis (AS). Canadian monograph recommended dosing of etanercept is $50 \mathrm{mg} / \mathrm{week}$, with select indications also requiring an initial loading phase of $100 \mathrm{mg} /$ week for 12 weeks. Evidence suggests that real-world practices differ from monograph, with patients titrating to lower or higher weekly dosing as needed. Limited research exists on how etanercept patients are dose optimized in the real-world Canadian setting.
Objectives: To describe etanercept treatment dynamics, including dose deescalation/escalation in the Canadian real-world setting.

Methods: A retrospective cohort study was conducted utilizing claims-level data from QuintilesIMS Private Drug Plan database, Ontario Public Drug Plan database, and Quebec Public Drug Plan database. Between 07/2013-06/2015, bio-naïve patients who initiated etanercept and who retained on therapy for 12 months were identified. Weekly dosing of each patient was calculated and analyzed for the prevalence and magnitude of dose de-escalation/escalation. Patients with at least $20 \%$ lower/higher average dose than monograph recommended dose (50mg/week) were flagged as dose de-escalators/dose escalators, respectively. The first 3 claims of etanercept were excluded from average dose calculations to exclude a possible loading phase.

Results: The study identified 3,051 etanercept patients $(60 \%$ female, $77 \%$ aged between 18 and $65,87 \%$ rheumatic diseases, and 13\% PsO) across Canada in the selection period. Overall, $11 \%(n=332)$ of patients de-escalated during their first year of therapy, led by AS $(15 \%, n=24)$ and RA $(12 \%, n=286) ; 15 \%$ $(n=449)$ of patients escalated, led by PsO $(64 \%, n=262)$ versus $7 \%(n=168)$ in RA; $74 \%(n=2,270)$ of patients maintained a consistent dose. Average dosing across rheumatic disease patients stabilized to monograph levels by week 20 of their therapy; PsO patients' dosing was observed to be lower than monograph during the loading phase, while higher than monograph in the maintenance phase.

Conclusions: In Canadian real-world practice, the average patient utilization of etanercept remained consistent over the first year in majority of patients, with the exception of those with $\mathrm{PsO}$. A notable proportion of etanercept patients with rheumatic diseases reduced their average dosing over time while on therapy, with almost twice as many patients titrating their dose downwards than upwards. However, in $\mathrm{PsO}$ patients, a majority of patients increased their etanercept.

Disclosure of Interest: M. Khraishi Consultant for: Pfizer Canada and Amgen Canada, Y. Zhang: None declared, J. Ivanovic: None declared, B. Millson: None declared, E. Singh: None declared, J. Woolcott: None declared, H. Jones: None declared

DOI: 10.1136/annrheumdis-2017-eular.6959

\section{THU0629 THE PERSPECTIVES OF PATIENTS, THEIR FIRST DEGREE RELATIVES, AND RHEUMATOLOGISTS AROUND PREVENTATIVE TREATMENTS FOR RHEUMATOID ARTHRITIS}

L. Spooner ${ }^{1}$, K. Milbers ${ }^{2}$, S. Munro ${ }^{1}$, N. Tsao ${ }^{1}$, M. Hudson ${ }^{3}$, C. Koehn ${ }^{4}$, N. Bansback ${ }^{1}$, M. Harrison ${ }^{1,2}$. ${ }^{1}$ University of British Columbia; ${ }^{2}$ Centre for Health Evaluation and Outcome Sciences, Vancouver; ${ }^{3}$ McGill University, Montreal; ${ }^{4}$ Arthritis Consumer Experts, Vancouver, Canada

Background: Ongoing randomized controlled trials are looking at the efficacy of preventing RA through treatment with anti-rheumatic medications. However even if these trials are successful, uncertainty around the potential benefits of preventative programs in practice will affect the uptake of a preventative treatment program. The views of rheumatologists, patients with RA and people considered at-risk of RA (potential recipients) will be important to consider.

Objectives: To identify relevant attributes for a discrete choice experiment (DCE) representing the factors that influence the preferences of patients, first-degree relatives (FDRs), and rheumatologists about preventative treatment for rheumatoid arthritis (RA).

Methods: Semi-structured focus groups were conducted with 1) RA patients in British Columbia (BC), Canada 2) FDRs of people with RA, and 3) rheumatologists from across Canada. Participants were recruited through a combination of convenience and homogenous sampling. Focus group guides were adapted from a previous study which used a DCE to represent an RA treatment decision. In the first round of focus groups, moderated discussions with RA patients, FDRs, and rheumatologists elicited open-ended responses to the interview guide questions. Findings from analysis of these discussions were reduced to a list of potential attributes for the DCE. In the second round, RA patients and FDRs provided feedback to improve the validity and representation of the potential attributes. All focus groups were audio recorded, transcribed, and analyzed using Framework Analysis. Results: Five focus groups were conducted with 13 RA patients, 5 FDRs, and 7 rheumatologists from four Canadian provinces. Analysis of the discussions revealed that all groups considered competing risks when considering a preventative treatment decision: risks of developing RA and when it might occur; accuracy of predictive tests and the risk of a false positive; and the risks of treatment itself. For rheumatologists, the empirical evidence supporting preventative tests and preventative treatments for RA, as well as treatment side effects were of significant importance. Interestingly, some rheumatologists did not consider prevention to be part of their role. FDRs frequently mentioned the impact that a preventive treatment would have on their lifestyle, the accuracy of predictive test, and weighing the potential benefits against side effects of treatment as key factors in decisions to take a preventative treatment. The health care provider's (nurse/family physician/rheumatologist) knowledge of RA and perceived trustworthiness was also important to FDRs in considering a heath care provider's recommendation for preventative treatment.

Conclusions: Our Framework Analysis highlighted key themes in this discussion which informed the attributes to be included in a DCE. Our findings suggest there are important differences in how patients, FDRs and rheumatologists value the uncertainties surrounding the potential benefits of a preventative treatment for RA. 
Acknowledgements: This work was supported by a grant from the Canadian Rheumatology Association through the Canadian Initiative for Outcomes in Rheumatology Care (CIORA).

Disclosure of Interest: None declared

DOI: 10.1136/annrheumdis-2017-eular.2007

\section{THU0630 OPIOID USE IN PATIENTS WITH POLYMYALGIA RHEUMATICA}

M.D. Richter $^{1}$, S.J. Achenbach ${ }^{2}$, J.A. Zamora-Legoff ${ }^{3}$, C.S. Crowson ${ }^{2}$, E.L. Matteson ${ }^{3}$. ${ }^{1}$ Graduate Medical Education; ${ }^{2}$ Division of Biomedical Statistics and Informatics; ${ }^{3}$ Division of Rheumatology, Mayo Clinic, Rochester, United States

Background: Polymyalgia rheumatic (PMR) is a systemic rheumatic inflammatory disease characterized primarily by musculoskeletal pain and stiffness. Glucocorticoid treatment is the current standard of care however the need for additional pain management, in particular the need for opioid therapy, has not been studied. Objectives: To examine the trends of chronic opioid use in patients with PMR over an 11 year period in Olmsted County, Minnesota, USA and compare this to subjects without the disease.

Methods: Retrospective data on opioid prescriptions were collected from 2005 to 2015 in a population-based incidence cohort of patients meeting the 2012 American College of Rheumatology classification criteria for PMR alongside comparison subjects. Poisson regression methods were used to compare opioid use between these groups.

Results: 244 patients with PMR and 211 non-PMR comparator subjects were included in the study. Rates of chronic opioid use were not significantly different between the two groups. $7.5 \%$ of patients with PMR were identified as chronic users by the end of the study period compared with $5.2 \%$ of non-PMR subjects. Any opioid use was also not significantly higher in PMR, with relative risk of $1.10(95 \% \mathrm{Cl} 0.97,1.26, \mathrm{p}=0.14)$. There were higher rates of chronic use among patients over 80 years in both groups.

Conclusions: PMR does not appear to be associated with increased rates of opioid use when compared with the general population.

Acknowledgements: This study was made possible by the Rochester Epidemiology Project, which receives support from the National institute on Aging of the $\mathrm{NIH}$.

Disclosure of Interest: None declared

DOI: 10.1136/annrheumdis-2017-eular.1237

\section{THU0631 DIRECT FINANCIAL BURDEN OF RHEUMATOID ARTHRITIS ON PATIENTS' LIFE IN A DEVELOPING NATION OF PAKISTAN, ONE YEAR PROSPECTIVE STUDY}

M.S. Ahmad. Orthopedic Surgery, Fazle Omar Hospital, Chenab Nagar, Pakistan

Background: Rheumatoid Arthritis is chronic inflammatory disease. Early diagnosis and management is necessary to ovoid joint destruction and to minimize disability. It effects 0.5 to 1 percent population. The female to male ratio is 3 to 1 . Health care cost is of great concern to patients, physicians and health care policy makers. The financial impact of rheumatoid arthritis treatment like any chronic illness is of great significance in developing society like Pakistan where 30 percent population lives below poverty line and annual per capita incomes are very low. Whereas prevalence is same with high medicines prices with poorly developed health insurance system and government funded hospitals are scanty.

Most of times attention in the field of health economy is focused on direct medical in general and hospital cost in particular but however there are some social societal customs which also increases the burden of direct cost. The direct cost includes expenditures like physician visit cost, diagnostic tests, medications. There are also some hidden charges to this direct cost which are not included in studies like transport charges, food bills during hospital stay and accompany person, female are companied by males. All included puts strain on economy and major share of annual income of patient is paid on management of disease. The worst scenario is when patient stops treatment and if lucky gets support from other sources like patient welfare societies or from relatives.

Objectives: To assess the direct cost of patient's every visit to hospital outpatient department prospectively for one year and total cost of one year was summed up and percentage to annual income spend on treatment was calculated.

Methods: Study carried out from January to December 2015 at Fazle Omar Hospital Rabwah Chenab Nagar and 150 patients either newly diagnosed or already on treatment of rheumatoid Arthritis were included. Data collected for next 1 year for each visit, patients with any other disease along with rheumatoid were excluded. The estimated annual income of patient or the person bearing the expenses was recorded before start of study. Every time patient visit hospital outpatient department, prescription copy kept and cost of medicines, laboratory investigations and consultation fee is calculated and a questionnaire is filled how much it cost coming hospital and going back home and during hospital stay on any other things. The total cost of every visit was recorded for one year.

Results: The mean total per patient income after conversion from local currency was 3000 US dollars against 1474 US dollars per capita income in 2015 . The annual average cost per patient including consultation fee, medicines purchased, laboratory investigations and other overhead expenses like transportation of patient and accompanied person and food bills during hospital stay was 1194 US dollars.
$41 \%$ of patient gross income was spend on management of rheumatoid arthritis. Investigations cost $12 \%$, medicines purchased $16 \%$, consultation $2 \%$ and over head visit charges cost $10 \%$ of the total mean of per patient annual income.

Conclusions: RA management consumes large portion of patients annual income and it has significant burden on developing world economy. References:

[1] The economic burden of rheumatoid arthritis in a developing nation: result from one year prospective cohort study in Thailand, Osiri M, Maetzel A, Tugwell P. J Rheumatol 2007 Jan.34(1):57-63.

Disclosure of Interest: None declared

DOI: 10.1136/annrheumdis-2017-eular.5571

\section{THU0632 VALIDATION OF INTERNET-BASED REPORTING OF PATIENT REPORTED OUTCOMES WITHIN THE SWEDISH RHEUMATOLOGY QUALITY REGISTER}

O.E. Hofstedt ${ }^{1}$, D. Di Giuseppe ${ }^{2}$, G.-M. Alenius ${ }^{1}$, N. Stattin ${ }^{3}$

$\mathrm{H}$. Forsblad-d'Elia ${ }^{1}$, L. Ljung ${ }^{1}$. ${ }^{1}$ Department of Public Health and Clinical Medicine, Umeå University, Umeå: ${ }^{2}$ Department of Medicine Solna, Karolinska Institutet, Stockholm; ${ }^{3}$ Swedish Rheumatism Association, Sundsvall, Sweden

Background: Previous studies have validated the use of clinic based touchscreens for registering patient reported outcome measures (PROMs) (1-3). The Swedish Rheumatology Quality (SRQ) register has implemented an internetbased method (PER (Patientens Egen Registrering, or Patients' sElf Registration)) for collecting PROMs.

Objectives: The aim of this study was to investigate the feasibility of the internet-based method as well as the validity of reported outcomes and disease activity scores compared to the gold standard paper format.

Methods: We recruited patients $(n=44$, mean age $=51.0$, standard deviation $=13.2$ years, $69.6 \%$ women) included in SRQ with a diagnosis of rheumatoid arthritis, psoriatic arthritis, juvenile arthritis, spondyloarthritis or ankylosing spondylitis. Before a planned visit at the rheumatology clinic the patients registered Visual Analog Scales (VAS) for global health, pain and fatigue, both electronically by PER and on paper. Patients with axial disease also registered BASDAI and BASFI related variables $(n=6)$. For patients with peripheral arthritis $(n=38)$, DAS28 was calculated using both methods. The differences between the methods were compared by T-test and Intra-class correlation (ICC). Agreement was visualized using Bland-Altman plots for all VAS registrations. The patients also answered a questionnaire regarding the used device and preferred method.

Results: No differences between PER or paper based VAS scores were found for VAS Global, VAS Pain and VAS Fatigue $(p=0.086, p=0.691$ and $p=0.197$, respectively). ICC scores ranged from 0.930 to 0.971 . Bland-Altman plots for VAS assessments showed good agreement and no proportional bias was detected (Fig 1). Mean difference for DAS28 was - 0.04 ( $p=0.177$ ). Of the recruited patients, $78 \%$, preferred the Internet based method. BASDAI and BASFI could not be evaluated due to a limited number of observations.

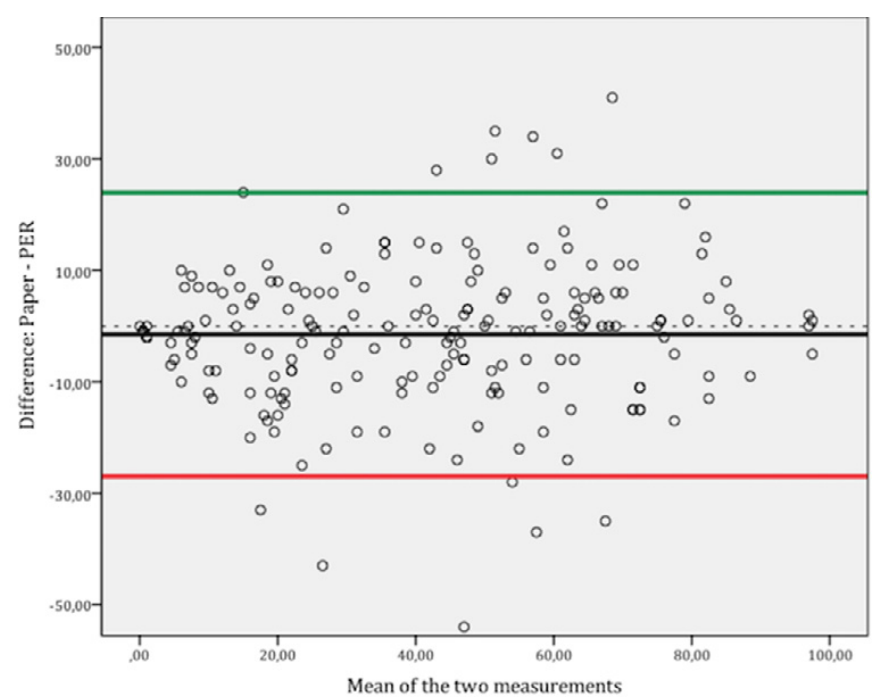

Fig. 1 Bland-Altman plot showing level of agreement of all VAS (VAS Global, VAS Pain, VAS Fatigue, VAS included in BASFI and BASDAI) between paper and PER method. BASDAI and BASFI scores multiplied by 10 for comparability.

Conclusions: Internet based reporting of PROMs supply valid VAS data. DAS28 scores from the internet-based method presents an acceptable alternative to the traditional paper formats.

\section{References:}

[1] Greenwood MC, et al. Rheumatology (Oxford). 2006

[2] Schefte DB, et al., Rheumatology (Oxford). 2010.

[3] Cunha-Miranda L, et al Rheumatol Int. 2015 